\title{
Reversible Watermarking Algorithm with Distortion Compensation
}

\author{
Vasiliy Sachnev, ${ }^{1}$ Hyoung Joong Kim, ${ }^{2}$ Sundaram Suresh, ${ }^{3}$ and Yun Qing Shi ${ }^{4}$ \\ ${ }^{1}$ School of Information, Communications, and Electronic Engineering, The Catholic University of Korea, \\ Bucheon 420-743, Republic of Korea \\ ${ }^{2}$ CIST, Korea University, Seoul 136-701, Republic of Korea \\ ${ }^{3}$ School of Computer Engineering, Nanyang Technological University, Singapore 639798 \\ ${ }^{4}$ Department of Electrical and Computer Engineering, NJIT, Newark, NJ 07102, USA \\ Correspondence should be addressed to Hyoung Joong Kim, khj-@korea.ac.kr
}

Received 8 September 2010; Accepted 14 December 2010

Academic Editor: Ling Shao

Copyright (C) 2010 Vasiliy Sachnev et al. This is an open access article distributed under the Creative Commons Attribution License, which permits unrestricted use, distribution, and reproduction in any medium, provided the original work is properly cited.

\begin{abstract}
A novel reversible watermarking algorithm with two-stage data hiding strategy is presented in this paper. The core idea is two-stage data hiding (i.e., hiding data twice in a pixel of a cell), where the distortion after the first stage of embedding can be rarely removed, mostly reduced, or hardly increased after the second stage. Note that even the increased distortion is smaller compared to that of other methods under the same conditions. For this purpose, we compute lower and upper bounds from ordered neighboring pixels. In the first stage, the difference value between a pixel and its corresponding lower bound is used to hide one bit. The distortion can be removed, reduced, or increased by hiding another bit of data by using a difference value between the upper bound and the modified pixel. For the purpose of controlling capacity and reducing distortion, we determine appropriate threshold values. Finally, we present an algorithm to handle overflow/underflow problems designed specifically for two-stage embedding. Experimental study is carried out using several images, and the results are compared with well-known methods in the literature. The results clearly highlight that the proposed algorithm can hide more data with less distortion.
\end{abstract}

\section{Introduction}

Data embedding techniques modify and, hence, distort the host signal (e.g., pixel values of image) in order to hide additional information. In many applications such as legal or medical images, loss of signal fidelity is undesirable. Hence, we need to develop reversible data hiding techniques, where the original host signal and the embedded message are able to be recovered exactly. In addition, these methods should have a high embedding capacity with less distortion. In general, high embedding capacity results in a high degree of distortion. Hence, these conflicting requirements stimulate interest among researchers in developing reversible watermarking algorithms with high capacity and low distortion. This paper addresses a novel approach by employing a twostage embedding strategy to achieve the goal.

The first reversible data hiding approach was presented by Mintzer et al. [1]. They proposed a visible embedding technique exploiting the property of reversibility of the original image. Fridrich et al. [2] used a lossless compression algorithm for reversible data hiding. Van der Veen et al. [3] proposed a companding technique for audio signals. Leest et al. [4] extended this technique for images. Celik et al. [5] proposed an LSB substitution technique using an efficient entropy coder. Yang et al. [6] utilized an integer discrete cosine transform (DCT). Yang et al. [7] exploited a histogram expansion technique for embedding data to high-frequency coefficients of the integer discrete wavelet transform (DWT). Xuan et al. [8-10] proposed several reversible data hiding techniques based on integer DWT. Zou et al. [11] proposed a semifragile reversible data hiding technique based on integer DWT. These improvements over reversible data hiding techniques were attained by reducing location map size or side information [12-14] or by using a new data hiding technique, such as difference expansion (DE) [15], improvement of (DE) [16, 17], companding [3, 
4], and histogram shifting [18, 19], and by using appropriate domain for data hiding, such as integer DCT [6], integer DWT $[7-10,20]$, and prediction errors $[14,19,21]$. The above-mentioned methods can be improved further.

In difference expansion [15], the image is divided into pairs of neighboring pixels. The difference between two pixel values in a pair is used for data hiding. Two kinds of overlapping problems arise after data hiding into pairs: (a) overlapping due to difference expansion (i.e., modified pairs are mixed with unmodified pairs) and (b) overlapping due to overflow/underflow (i.e., some pairs cannot be modified). These overlapping problems are solved by marking all pairs in the location map. The location map must be compressed and added to the original payload.

The biggest problem in the original difference expansion method is the huge size of the location map. Even after compression, the location map occupies a significant portion of the payload. Thus, decreasing the size of the location map has been a challenging problem. Many improvements $[12,13,22,23]$ over the Tian's difference expansion aim to decrease the location map size. Kamstra and Heijmans [12] improved the difference expansion method by sorting pairs according to correlation factors computed using average values of the neighboring pairs. The location map covers only a portion of the sorted pairs, which contributes to the increase in payload. The compact location map achieves higher capacity but produces a similar level of distortion. Kim et al. [13] decreased the size of the location map further by removing nonambiguous parts. Their method [13] achieves better results when compared to Kamstra and Heijmans' method [12]. All these expansions increase the payload but fail to minimize the distortion significantly.

$\mathrm{Ni}$ et al. [18] used a histogram shifting technique in the spatial domain. Thodi and Rodríguez [19] explored the histogram shifting method by employing the prediction errors for efficiency. Sachnev et al. [21] improved the performance of the prediction error expansion by using sorting. Exploiting the histogram shifting approach to JPEG-LS prediction errors produces excellent results. The histogram shifting approach solves an overlapping problem by using the location map covered only prediction errors which can possibly cause overflow/underflow errors after data hiding. As a result, the histogram shifting method significantly decreases the location map size and sometimes can also eliminate the necessity of it.

Lee et al. [20] used an advanced watermarking technique based on integer-to-integer wavelet transform. Their method divides image into nonoverlapping blocks and applies a data hiding technique based on the definitions of expandability (which means a possibility of bit shifting operation) and changeability over high-frequency wavelet coefficients of each block. Bit-shifting approach is used for embedding data, and an LSB replacement approach for hiding the location map. Expanded and nonexpanded blocks are marked by different flags, 1 or 0 , respectively, in the location map. It covers all blocks, and its size is $(X / N) \times(Y / M)$, where $N$ and $M$ are the block size, and $X$ and $Y$ are the image size. In order to achieve reversibility, the proposed method requires location map, expansion matrix $P$, and original LSB of coefficients from the blocks containing location map. The proposed technique outperforms existing methods as $[2,7$, $8,22]$ by exploiting high-frequency subbands and efficient data hiding technique. Even though the performance is better than $[2,7,8,22]$, the requirement of location map, original LSB of coefficients from the blocks containing location map, and expansion matrix influence the capacity of the method.

This paper presents a new two-stage embedding strategy which hides more data with lower distortion compared to the existing reversible data hiding methods. In the proposed scheme, two bounds based on neighboring pixels are used to possibly hide data twice in a given pixel. First, the neighboring pixels are ordered, and the lower and upper bounds are calculated. The difference value between a pixel and its lower bound is used for hiding one bit according to the rules of histogram shifting. Next, the difference between the upper bound and the modified pixel is used for hiding another bit, such that the distortion in the first stage can be possibly reduced. Due to heterogeneity in the image's features, the proposed strategy may remove at rare occasions, mostly reduce, or hardly increase distortion after the second stage of embedding. In this paper, we show the efficiency of the proposed method by calculating the portioned distortion impact of different scenarios (i.e., the case of removing, reducing, or increasing distortion), and by highlighting the theoretical efficiency compared with histogram shifting for the same conditions. Finally, we present the experimental results with comparison of the performance with wellknown methods for four most popular test images. The results clearly illustrate its high capacity with low distortion.

The organization of this paper is as follows. Section 2 explains the rationale of using two-stage embedding. Section 3 discusses important issues regarding the proposed method including two-stage embedding in detail, different scenarios in two-stage embedding, and a solution for overflow and underflow problems. Section 4 describes the encoder and decoder of the proposed method. Section 5 presents the experimental results. Section 6 concludes the paper.

\section{Rationale of Using Two-Stage Embedding}

Some reversible data hiding methods [13-15, 22-24] use the concept of difference expansion transform. The difference expansion transform is based on a reversible integer Haar wavelet transform. Another approach commonly used in reversible data hiding is histogram shifting over prediction errors [19]. In all these schemes, the expansion affects the image quality. In this section, we first present the motivation of our work by highlighting the issues in expansion strategy. Later, we will present a strategy that can reduce distortion.

The well-known difference expansion method (DE) [15] uses the difference value between two neighboring pixels for hiding one bit of data. For a given pair of pixels $(x, y), x, y \in$ $Z, 0 \leq x, y \leq 255$, the difference expansion methods embed one bit of data $b, b \in[0,1]$ as follows:

$$
H=2 \cdot h+b,
$$


where $h$ is the difference value between pair of pixels $(x, y)$, and $H$ is the modified difference value after hiding data. The modified pair of pixels is $(X, Y)$, where $H=X-Y$.

The total distortion in a pair $(x, y)$ after data hiding is expressed as follows (see Figure 1(a)):

$$
D_{\mathrm{DE}}=|H-h|=|2 \cdot h+b-h|=|h+b| .
$$

The distortion of the prediction error expansion (PEE) can be calculated in the same fashion. Let $\hat{m}$ be a predicted pixel value of a pixel $m$, then $d=m-\hat{m}$ is the prediction error. The prediction error expansion hides one bit of data as follows (see Figure 1(b)):

$$
d^{\prime}=2 \cdot d+b .
$$

Hence, the distortion generated by prediction error expansion is

$$
D_{\mathrm{PE}}=\left|d^{\prime}-d\right|=|2 \cdot d+b-d|=|d+b| .
$$

Note that the distortion of the difference errors expansion and prediction errors expansion are similar in nature.

Rationale for New Strategy. From the above analysis, we can see that the distortion of both methods directly depends on the difference value $h$ or prediction error $d$. Hence, we need to find a suitable expansion technique, which has errors less then $|h|$ or $|d|$. The main objective of reversible watermarking is to find a method which can embed more data with less distortion. Hence, we present a new strategy. In this strategy, each pixel is possibly expanded twice by embedding two bits of data. For every pixel, the lower and upper bounds are computed from eight neighboring pixels. Let $a_{i}$ for $i=1,2, \ldots, 8$ be the surrounding pixels for a pixel $a_{0}$ as shown in Figure 2. The central pixel $a_{0}$ and its eight neighboring pixels define a cell for embedding data. The neighboring pixels are sorted in ascending order to calculate the lower and upper bounds as follows:

$$
\begin{aligned}
& L_{1}=\left\lfloor\frac{\sum_{n=1}^{4} a_{S_{n}}}{4}\right\rfloor, \\
& L_{2}=\left\lfloor\frac{\sum_{n=5}^{8} a_{S_{n}}}{4}\right\rfloor,
\end{aligned}
$$

where $a_{s_{n}}$ is a set of sorted neighboring pixels. The first stage of the proposed data hiding technique is represented as follows:

$$
\begin{gathered}
e_{1}=a_{0}-L_{1}, \\
E_{1}=2 \cdot e_{1}+b_{1}, \\
A_{1}=L_{1}+E_{1} .
\end{gathered}
$$

The distortion after the first stage of embedding is given as $D_{1}=\left|A_{1}-a_{0}\right|$. The second stage of the proposed data hiding technique is represented as follows:

$$
\begin{gathered}
e_{2}=L_{2}-A_{1}, \\
E_{2}=2 \cdot e_{2}+b_{2}, \\
A_{2}=L_{2}-E_{2} .
\end{gathered}
$$

The distortion after data hiding is

$$
\begin{aligned}
D_{2} & =\left|A_{2}-a_{0}\right| \\
& =\left|L_{1}-L_{2}+3 \cdot e_{1}+2 \cdot b_{1}-b_{2}\right| .
\end{aligned}
$$

Note that the resulted distortion $D_{2}$ depends on the utilized data embedding strategy. In our paper, we use the histogram shifting strategy for data hiding. Here, (7) and (10) depend on the differences $e_{1}$ and $e_{2}$, respectively. Such cases will be explained later in Section 3.1.

In the proposed strategy, we can embed two bits with less distortion compared to a single embedding. Assume that the first hidden bit $b_{1}$ is 1 , second hidden bit $b_{2}$ is $1, a=100$, $L_{1}=98, L_{2}=104$, and $e_{1}=2$. First stage of embedding gives $E_{1}=2 \cdot e_{1}+b_{1}=5$, the central pixel value $A_{1}=103$, and the distance value $e_{2}=L_{2}-A_{1}=104-103=1$. Note that the distortion $D_{1}=3$ after first stage of embedding is the same with distortion of DE and PEE (i.e., $e+b$ ). Second stage of embedding gives $E_{2}=2 \cdot e_{2}+b_{2}=3$, the central pixel $A_{2}=L_{2}-E_{2}=104-3=101$. The second stage of embedding reduces distortion from 3 (distortion after the first stage) to 1. The resulted distortion $D_{2}$ after hiding two bits of data is less than the distortion in DE and PEE for a single embedding (see Figure 1(b)).

In the next section, we present the proposed data hiding algorithm with all possible scenarios and their distortion.

\section{Two-Stage Embedding Algorithm Using Histogram Shifting}

In the proposed scheme, we can embed data twice with possibly reduced distortion. As explained in the previous section, first we calculate $L_{1}$ and $L_{2}$ using the sorted neighboring pixels. For data hiding in each stage, we use the modification of the histogram shifting technique proposed by Thodi and Rodríguez [19]. For the proposed data hiding technique, we suggest an algorithm to find the appropriate threshold values $T_{n}$ and $T_{p}$ (i.e., negative and positive) similar to the original method. Now, we present the steps required to encode and decode the hidden data using a twostage embedding technique.

Encoding. The algorithm embeds data $\left(b_{1}, b_{2}\right)$ in two stages. First, the first bit $b_{1}$ is hidden using $L_{1}$, and next the second bit $b_{2}$ is hidden using $L_{2}$.

First stage:

$$
E_{1}= \begin{cases}2 \cdot e_{1}+b_{1}, & \text { if } e_{1} \in\left[T_{n} ; T_{p}\right], \\ e_{1}+T_{p}+1, & \text { if } e_{1}>T_{p}, \\ e_{1}+T_{n}, & \text { if } e_{1}<T_{n},\end{cases}
$$

where $e_{1}=a_{0}-L_{1}$.

Note that the expandable set is $E=e \in\left[T_{n} ; T_{p}\right]$ and the shiftable set is $S=e \in\left(-\infty ; T_{n}\right) \cup\left(T_{p} ; \infty\right)$.

The pixel value $a_{0}$ after embedding $b_{1}$ is changed to

$$
A_{1}=L_{1}+E_{1} \text {. }
$$




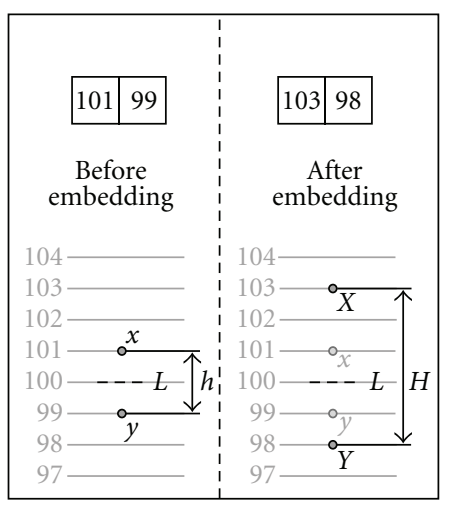

(a)

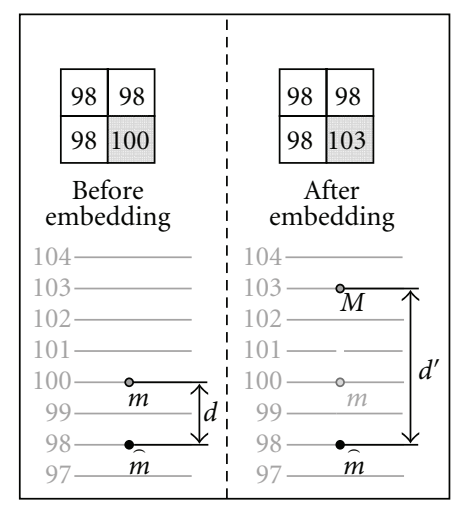

(b)

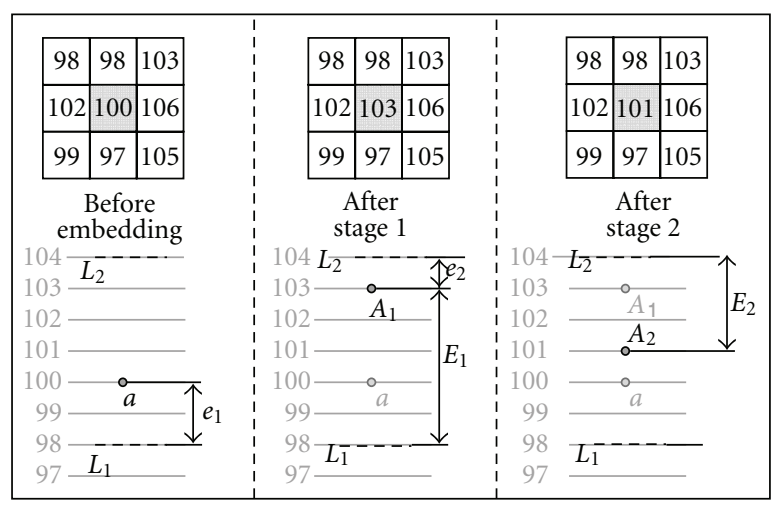

(c)

Figure 1: Different expansion strategies ((a) DE; (b) PEE; (c) Two-stage embedding).

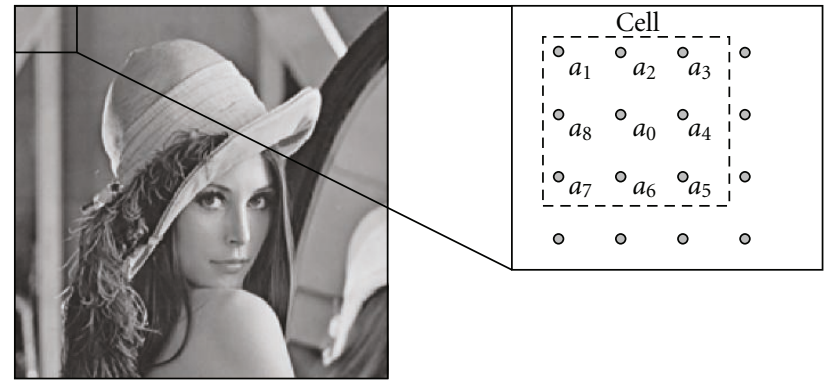

Figure 2: A cell for the proposed scheme.

Second stage: Now, we hide the second bit of data $b_{2}$ in $A_{1}$ using $L_{2}$. The embedding process is designed as one

$$
E_{2}= \begin{cases}2 \cdot e_{2}+b_{2}, & \text { if } e_{2} \in\left[T_{n} ; T_{p}\right], \\ e_{2}+T_{p}+1, & \text { if } e_{2}>T_{p}, \\ e_{2}+T_{n}, & \text { if } e_{2}<T_{n},\end{cases}
$$

where $e_{2}=L_{2}-A_{1}$. follows:

The pixel value $A_{1}$ after embedding $b_{2}$ is represented as

$$
A_{2}=L_{2}-E_{2}
$$

$T_{p}$ and $T_{n}$ are the positive and negative threshold values. The threshold values can be approximately obtained using the histogram of the $e_{1}$. Assume that the first and second stages of embedding have the same payload, and the histogram's shape of $e_{1}$ and $e_{2}$ is similar. Thus, for the given payload $P$, the approximate threshold values $T_{p}$ and $T_{n}$ are chosen such that $|E|>0.5 \cdot|P|$, where $E=e_{1} \in\left[T_{n} ; T_{p}\right]$, and $|E|$ is the number of elements in the set $E$. In reality, due to difference between histogram's shape of $e_{1}$ and $e_{2}$ (here, note that the exact value $e_{2}$ can be computed only after hiding data to $e_{1}$ ), the approximate threshold values may not be large enough to hide the payload $P$. Thus, if that happens, the magnitudes of the threshold values have to be increased, and the embedding process has to be repeated

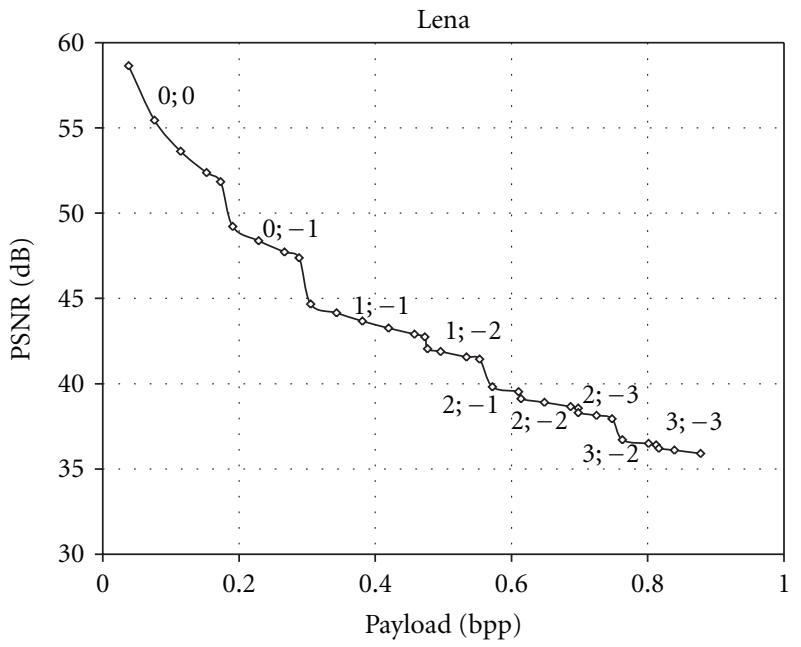

Figure 3: Appropriate threshold values for Lena image.

with new threshold values. Note that the proposed algorithm can exactly predict the threshold values for the first stage of embedding. Thus, the approximate threshold values have the minimal possible magnitudes to hide a necessary payload. We test the proposed algorithm and find that for most of payloads the approximate threshold values are suitable for data hiding. When the payload is large $(\approx 1 \mathrm{bpp})$, the proposed algorithm requires one more iteration. In case of extreme payloads $(\approx 1.5 \mathrm{bpp})$ close to the maximum possible size (see Figure 7), the proposed algorithm requires multiple iterations. In Figure 3, we illustrate the appropriate threshold values for different payloads computed using the proposed method. If the payload approaches to the point to be increased (i.e., at $0.18 \mathrm{bpp}, 0.29 \mathrm{bpp}$, or $0.47 \mathrm{bpp}$ ), the proposed method updates the threshold values (see Figure 3).

Note that, in general, the threshold values for two-stage embedding have lower magnitude compared to the histogram shifting method (see Table 1) due to high embedding capacity, which results in lower distortion in image. For example, in case of hiding $120 \mathrm{kbits}$ of data to Lena image, 
the threshold values for histogram shifting are -2 and 2, while for the proposed method they are -1 and 1 . Such low threshold values help in improving capacity and low distortion.

In the decoding process, the lower and upper bounds calculated using the neighboring pixels remain the same as in the encoder. Now, we present the steps required to decode the hidden data.

Decoding. Let $\left(A_{2}, a_{1}, a_{2}, \ldots, a_{8}\right)$ in a single cell be used for decoding data. The $L_{1}$ and $L_{2}$ are calculated using the sorted neighboring pixels as described in (5).

First stage: the decoding process can be described as

$$
e_{2}= \begin{cases}\left\lfloor\frac{E_{2}}{2}\right\rfloor & \text { if } E_{2} \in\left[2 \cdot T_{n} ; 2 \cdot T_{p}+1\right], \\ E_{2}-T_{p}-1, & \text { if } E_{2}>2 \cdot T_{p}+1, \\ E_{2}-T_{n}, & \text { if } E_{2}<2 \cdot T_{n},\end{cases}
$$

where $E_{2}=L_{2}-A_{2}$.

The second hidden bit $b_{2}$ is retrieved using

$$
b_{2}=E_{2} \bmod 2, \quad E_{2} \in\left[2 \cdot T_{n} ; 2 \cdot T_{p}+1\right] .
$$

After retrieving the data from the pixel value $A_{2}$, the pixel $A_{1}$ is computed as follows:

$$
A_{1}=L_{2}-e_{2}
$$

Second stage: now, we retrieve the first data $b_{1}$ from $A_{1}$. The decoding process is defined as follows:

$$
e_{1}= \begin{cases}\left\lfloor\frac{E_{1}}{2}\right\rfloor & \text { if } E_{1} \in\left[2 \cdot T_{n} ; 2 \cdot T_{p}+1\right], \\ E_{1}-T_{p}-1, & \text { if } E_{1}>2 \cdot T_{p}+1, \\ E_{1}-T_{n}, & \text { if } E_{1}<2 \cdot T_{n},\end{cases}
$$

where $E_{1}=A_{1}-L_{1}$.

The first hidden bit $\left(b_{1}\right)$ is retrieved using

$$
b_{1}=E_{1} \bmod 2, \quad E_{1} \in\left[2 \cdot T_{n} ; 2 \cdot T_{p}+1\right] .
$$

The original pixel value $a_{0}$ after retrieving $b_{1}$ is recovered as follows:

$$
a_{0}=L_{1}+e_{1} .
$$

The total distortion of the proposed two-stage embedding is $D_{2}=A_{2}-a_{0}$, where $A_{2}$ is computed using (16). Here, the modified pixel $A_{2}$ depends on the different scenarios in the (13) and (15).

Thus, for $e_{1} \in\left[0 ; T_{p}\right]$ (expandable, hiding bit $b_{1}$ ) and $e_{2} \in\left[T_{n} ; T_{p}\right]$ (expandable, hiding bit $b_{2}$ ), we have

$$
D_{2}=L_{1}-L_{2}+3 \cdot e_{1}+2 \cdot b_{1}-b_{2} .
$$

For $e_{1}>T_{p}$ (shiftable, shifting by $\left.T_{p}\right)$ and $e_{2} \in\left[T_{n} ; T_{p}\right]$ (expandable, hiding bit $b_{1}$ ), we have

$$
D_{2}=L_{1}-L_{2}+e_{1}+2 \cdot T_{p}+2-b_{1} .
$$

For $e_{1}>T_{p}$ (shiftable, shifting by $T_{p}$ ) and $e_{2}<T_{n}$ (shiftable, shifting by $T_{n}$ ), or $e_{1}<T_{n}$ (shiftable, shifting by $T_{n}$ ) and $e_{2}>$ $T_{p}$ (shiftable, shifting by $T_{p}$ ), we have

$$
D_{2}=T_{p}-T_{n}+1
$$

For $e_{1} \in\left[T_{n} ; T_{p}\right]$ (expandable, hiding bit $b_{1}$ ) and $e_{2}>T_{p}$ (shiftable, shifting by $T_{p}$ ), we have

$$
D_{2}=e_{1}+b_{1}-T_{p}-1
$$

Using the decoding process, we can retrieve the original pixel value $a_{0}$ and the hidden data $b_{1}$ and $b_{2}$. The main advantage of the proposed method is that the distortion due to data hiding in the first stage can be reduced in the second stage efficiently. Hence, we propose the two-stage embedding scheme to achieve high capacity with low distortion.

3.1. Different Scenarios in Two-Stage Embedding. The minimization of distortion due to the first stage at the second stage depends on $e_{1}$. Based on the value $e_{1}$, there exist three possible scenarios, namely: removable, half-removable, and nonremovable cases (see Figure 4).

Removable. In this scenario, the distortion due to the firststage embedding is removed completely in the second stage (i.e., $D_{2}=0$ ).

The equality for removing distortion is derived differently from (23), (24), (25), and (26).

For $e_{1} \in\left[0 ; T_{p}\right]$ (expandable, hiding bit $b_{1}$ ) and $e_{2} \in$ $\left[0 ; T_{p}\right]$ (expandable, hiding bit $b_{2}$ ), we have

$$
e_{1}=\left\lfloor\frac{L_{2}-L_{1}-2 \cdot b_{1}+b_{2}}{3}\right\rfloor \text {. }
$$

For $e_{1} \in\left[0 ; T_{p}\right]$ (expandable, hiding bit $b_{1}$ ) and $e_{2}>T_{p}$ (shiftable, shifting by $T_{p}$ ), we have

$$
e_{1}=T_{p}-b_{1}+1
$$

For $e_{1}>T_{p}$ (shiftable, shifting by $\left.T_{p}\right)$ and $e_{2} \in\left[0 ; T_{p}\right]$ (expandable, hiding bit $b_{1}$ ), we have

$$
e_{1}=L_{2}-L_{1}-2 \cdot T_{p}-2+b_{1} .
$$

For $e_{1}>T_{p}$ and $e_{2}>T_{p}$ (both shiftable, shifting by $T_{p}$ ), the distortion will be removed completely (i.e., $D_{2}=0$ ).

Note that for the $e_{1} \in\left[T_{n} ; 0\right)$ or $e_{2} \in\left[T_{n} ; 0\right)$, the distortion cannot be removed in nature.

Half-Removable. In this scenario, the distortion due to the first-stage embedding can be removed partially in the second-stage. The distortion can be reduced or remain the same. In this case, the modified pixel value $A_{1}$ should not be greater than the upper bound $L_{2}$. Thus, the difference between the upper bound and modified pixel value $A_{1}$ keeps the sign (i.e., $L_{2}-A_{1} \geq 0$ ). In this case, the second stage embedding will decrease overall distortion. This scenario will occur when $A_{1} \leq L_{2}$. 


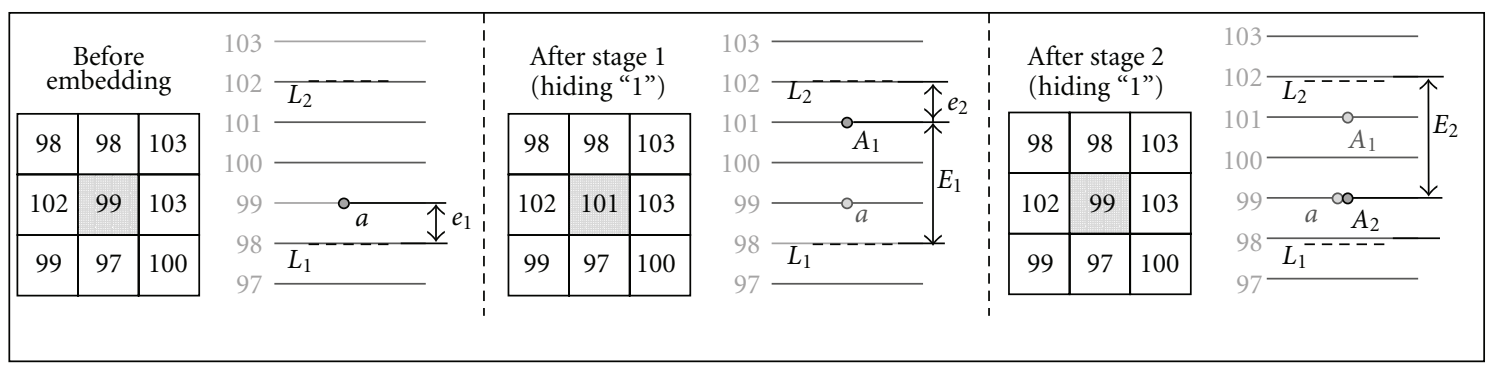

(a) Removable case

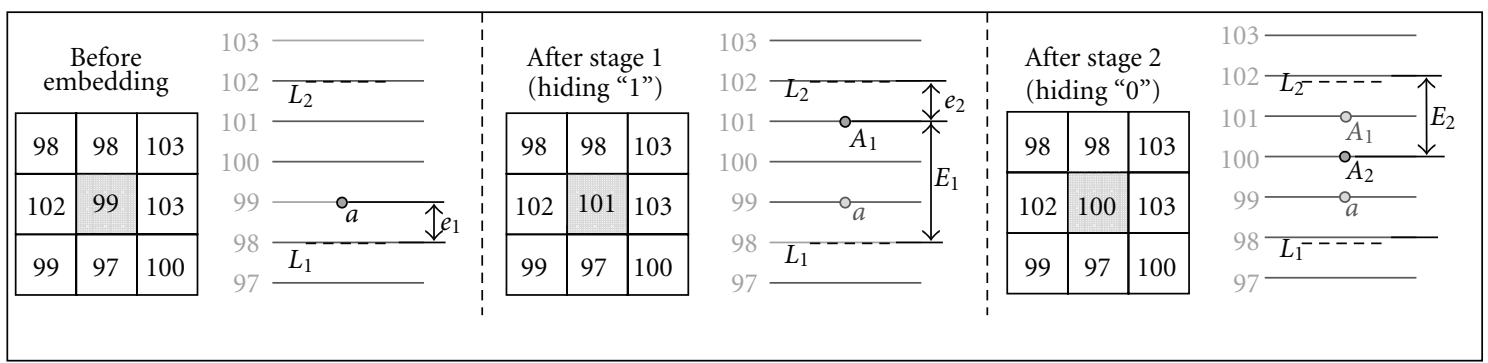

(b) Half-removable case

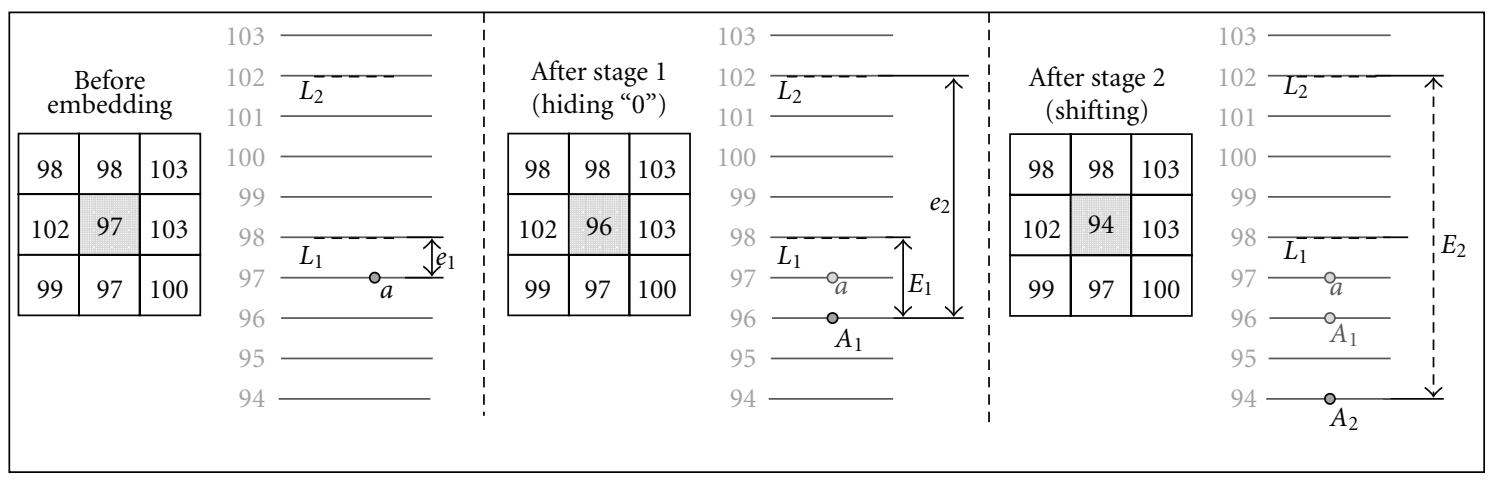

(c) Nonremovable case

FIgURE 4: Different scenarios of the two-stage embedding.

This inequality can be derived differently in respect of value $e_{1}$.

For $e_{1} \in\left[0 ; T_{p}\right]$, we have

$$
\begin{aligned}
A_{1} & \leq L_{2}, \\
L_{1}+2 \cdot e_{1}+b_{1} & \leq L_{2}, \\
e_{1} & \leq \frac{L_{2}-L_{1}-b_{1}}{2} .
\end{aligned}
$$

For $e_{1} \in\left[0 ; T_{p}\right]$ and $e_{2} \in\left[0 ; T_{p}\right]$ or $e_{2}>T_{p}$, the distortion $D_{2}$ is calculated using (23) or (26), respectively.

For $e_{1}>T_{p}$, we have

$$
\begin{aligned}
A_{1} & \leq L_{2}, \\
L_{1}+e_{1}+T_{p}+1 & \leq L_{2}, \\
e_{1} & \leq L_{2}-L_{1}-T_{p}-1 .
\end{aligned}
$$

Similarly, for the difference $e_{2} \in\left[0 ; T_{p}\right]$, the distortion $D_{2}$ is calculated using (24). Note that for the difference $e_{2}>T_{p}$, the distortion $\mathrm{D}_{2}$ becomes 0 (i.e., the cell belongs to the removable case).

Nonremovable. In this scenario, the distortion will increase after the second stage of embedding. This scenario occurs when $e_{1}<0$ or $A_{1}>L_{2}$.

The inequality $A_{1}>L_{2}$ can be rewritten similarly with the half-removable case.

For $e_{1} \in\left[0 ; T_{p}\right]$, we have

$$
e_{1}>\frac{L_{2}-L_{1}-b_{1}}{2} .
$$

In this case, for $e_{2} \in\left[T_{n} ; 0\right)$ or $e_{2}<T_{n}$, distortion $D_{2}$ is calculated using (23) or (25), respectively.

For $e_{1}>T_{p}$, we have

$$
e_{1}>L_{2}-L_{1}-T_{p}-1
$$

Similarly, for the difference $e_{2} \in\left[T_{n} ; 0\right)$ or $e_{2}<T_{n}$, the distortion $D_{2}$ is calculated using (24) or (25), respectively. 
Note that $D_{2}$ is always larger than $D_{1}$ for both $e_{1}<0$ and $e_{2}<0$.

From the above three scenarios, we can see that the proposed two-stage embedding strategy either removes, reduces, or increases the distortion. Similarly, the distortion in the proposed strategy depends on the selected threshold values. Since the proposed method can embed data twice, the selected threshold values for a given capacity is less than the threshold values for histogram shifting. From Table 1, we can see that the threshold values for the proposed method are 2550 percent lower. In some cases where the required payload is low, the threshold values are the same. Note that the distortion depends on the threshold values as well as the population of pixels (cells) that cause distortion. In the proposed two-stage embedding method, the cells of the different cases (i.e., removable, half-removable, and nonremovable) cause different distortion impact. The nonremovable cells do not cause distortion at all. The distortion of the half-removable cells after the double embedding in our method is less than a single embedding in DE or PEE. The nonremovable cells cause higher distortion than that of DE and PEE under the same thresholds. Thus, in order to estimate the performance of the proposed method we have to analyze the distortion impact of the different cells unified to the specific classes as removable, half-removable, and nonremovable for the proposed two-stage embedding method, and expandable and shiftable for the histogram shifting method.

3.2. Efficiency of the Two-Stage Embedding. The efficiency can be estimated numerically by computing the portioned distortion of the different cells for the two-stage embedding and the histogram shifting. Such an analysis may help evaluate the distortion impact of the removable, halfremovable, and nonremovable cells to the total distortion.

Since, the PSNR is the logarithmic measure of the MSE (see (34)), the distortion impact of different pixels (cells) can be calculated as an impact to the MSE,

$$
\mathrm{PSNR}=10 \cdot \log _{10}\left(\frac{255^{2}}{\mathrm{MSE}}\right)
$$

where MSE is the mean squared error,

$$
\mathrm{MSE}=\frac{1}{m \cdot n} \sum_{i=0}^{n-1} \sum_{j=0}^{m-1}\|I(i, j)-K(i, j)\|^{2}=\frac{1}{m \cdot n} \mathrm{SE},
$$

where $n, m$ are the height and width of the image, $I$ is the original image, $K$ is the modified image, and SE is the total squared error.

The total squared error (SE) can be calculated as follows:

$$
\mathrm{SE}=\mathrm{SE}_{0}+\mathrm{SE}_{1}+\mathrm{SE}_{2}
$$

where

$$
\begin{aligned}
\mathrm{SE}_{0}= & 0 \quad \text { if } I(i, j) \in \text { removable cells, } \\
\mathrm{SE}_{1}= & \sum \sum\|I(i, j)-K(i, j)\|^{2} \\
& \text { if } I(i, j) \in \text { half-removable cells, } \\
\mathrm{SE}_{2}= & \sum \sum\|I(i, j)-K(i, j)\|^{2} \\
& \text { if } I(i, j) \in \text { nonremovable cells. }
\end{aligned}
$$

From (35) and (36), derive the PSNR as follows:

$$
\mathrm{PSNR}=10 \cdot \log _{10}\left(\frac{m \cdot n \cdot 255^{2}}{\mathrm{SE}_{1}+\mathrm{SE}_{2}}\right)
$$

Thus, the total distortion (PSNR) can be estimated using the squared errors of all the half-removable cells $\left(\mathrm{SE}_{1}\right)$ and nonremovable cells $\left(\mathrm{SE}_{2}\right)$.

In our tests, we compare the squared errors (SE) and population of the half-removable and nonremovable cells (for the proposed method), and the expandable and shiftable cells (for the histogram shifting method). To illustrate the performance better, we study the squared error of the cells for Lena images versus the threshold values and payloads for Lena image. The results are reported in Table 1.

When the payload is $70 \mathrm{kbits}$, the proposed two-stage embedding method has 21,138 half-removable cells with squared error 21,102, and 77,151 nonremovable cells with squared error 266,157 . The total squared error is 287,259 , which causes PSNR $47.72 \mathrm{~dB}$. For the same payload, the histogram shifting method has 70,000 expandable cells with squared error 71,144 , and 166,113 shiftable cells with squared error 409,255 . The total squared error is 487,399 , which causes PSNR $45.25 \mathrm{~dB}$. Thus, when the payload is $70 \mathrm{kbits}$, the total squared error of the proposed method is 200,140 lower, and the PSNR is $2.47 \mathrm{~dB}$ higher. For larger payloads such as 120 and $150 \mathrm{kbits}$, the PSNR value of the proposed method is 1.31 and $0.83 \mathrm{~dB}$ higher, respectively. Hence, the PSNR value for the proposed method is better than that of the histogram shifting method.

3.3. Overflow and Underflow Problems. An important issue in data hiding is to avoid overflow or underflow errors where the modified pixels exceed the 8-bit range $[0 ; 255]$. These problematic pixels should be skipped from the embedding process. Such pixels are called skipped cells that can exceed the boundary (i.e., $A_{1}<0, A_{1}>255$ or $A_{2}<0$, $\left.A_{2}>255\right)$. Note that the skipped original cells and some modified cells which can cause overlapping with unmodified cells should be marked in the location map; otherwise, decoding will not be possible (refer to [19]). In this method, the decoder probes the embedding environment through the simulation. Note that the encoder does not modify the skipped cells which cause overflow/underflow. Thus, the simulation of the embedding process in the decoder has the overflow/underflow in the same cells as in the encoder. However, the simulation of embedding also causes overflow/underflow for some cells that were modified during data hiding. These overlapped cells have to be marked in 
TABLE 1: Populations of cells from different scenarios and sets versus different capacities for Lena image.

\begin{tabular}{|c|c|c|c|c|c|c|c|}
\hline \multirow{3}{*}{ Payload kbits } & \multicolumn{7}{|c|}{ Two-stage embedding } \\
\hline & \multirow{2}{*}{$T_{n} ; T_{p}$} & \multicolumn{2}{|c|}{ Half-removable case } & \multicolumn{2}{|c|}{ Nonremovable case } & \multirow{2}{*}{ Total SE } & \multirow{2}{*}{ PSNR $[\mathrm{dB}]$} \\
\hline & & Population & SE & Population & SE & & \\
\hline 70 & $-1 ; 0$ & 21,138 & 21,102 & 77,151 & 266,157 & 287,259 & 47.72 \\
\hline 120 & $-1 ; 1$ & 64,100 & 132,453 & 90,292 & 743,415 & 875,869 & 42.89 \\
\hline \multirow[t]{2}{*}{150} & $-1 ; 2$ & 69,290 & 277,756 & 111,507 & $1,500,101$ & $1,777,857$ & 39.82 \\
\hline & \multicolumn{7}{|c|}{ Histogram shifting [19] } \\
\hline \multirow{2}{*}{ Capacity kbits } & \multirow{2}{*}{$T_{n} ; T_{p}$} & \multicolumn{2}{|c|}{ Expandable set } & \multicolumn{2}{|c|}{ Shiftable set } & \multirow{2}{*}{ Total SE } & \multirow{2}{*}{ PSNR $[\mathrm{dB}]$} \\
\hline & & Population & SE & Population & SE & & \\
\hline 70 & $-1 ; 1$ & 70,000 & 78,144 & 166,113 & 409,255 & 487,399 & 45.25 \\
\hline 120 & $-2 ; 2$ & 120,000 & 277,651 & 141,098 & 906,021 & $1,183,672$ & 41.58 \\
\hline 150 & $-3 ; 3$ & 150,000 & 580,788 & 105,744 & $1,310,011$ & $1,890,799$ & 38.99 \\
\hline
\end{tabular}

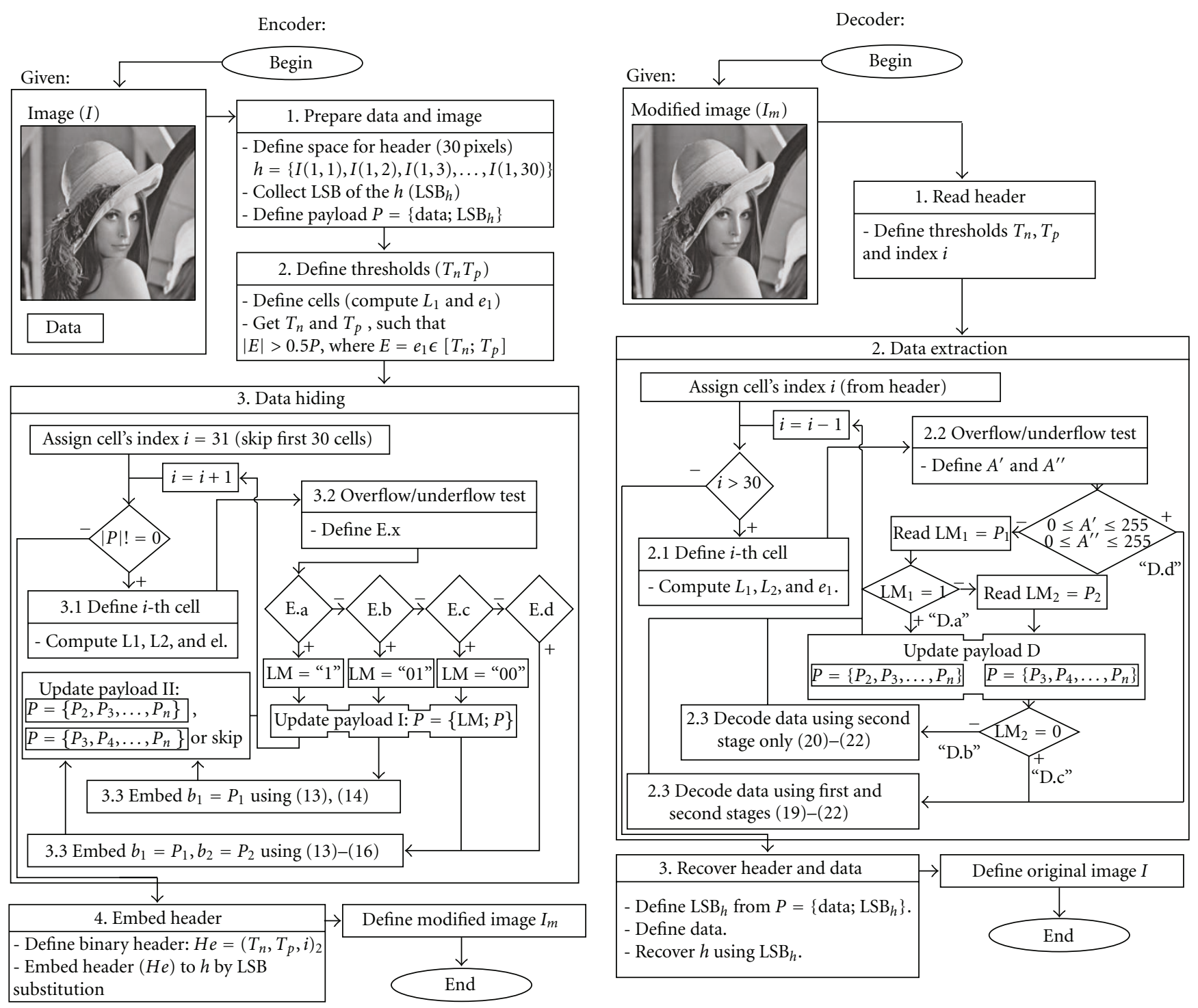

FIGURE 5: Flowchart of the encoder and decoder. 
the location map. Such a solution causes some additional problems. The decoder can not know the proper data hidden in the encoder side. Thus, the verification test (see below) in the encoder and decoder must use the same data. In our method this data is called "test bits." Note that hiding " 0 " causes distortion less than hiding " 1 " to the same cell for a positive difference value. Thus, sometimes hiding " 1 " causes overflow/underflow, but hiding " 0 ” does not. In this case, decoder does not know the proper data and may make wrong decision about the cell. Such a wrong decision is triggered by the wrong location map bits, that will cause the cascade misclassification for the rest of the location map and will also provide wrong recovered data. We solve this problem by adjusting the "test bits" as " 1 " for positive $e$ and " 0 " for negative.

Since the proposed method can hide two bits into a single cell, the problem of overflow/underflow can occur in any stage of embedding. Hence, we need one or two bits to identify the overlapping cells. There are four possible cases regarding the overflow/underflow problems for the encoder.

\subsubsection{Overflow/Underflow Test for Encoder}

Input. The cell for testing (i.e., $\left.\left(a_{0}, a_{1}, a_{2}, \ldots, a_{8}\right)\right)$; data to hide $b_{1}$ and $b_{2}$; threshold values $T_{n}$ and $T_{p}$.

Output. Case of the cell (i.e., E.a, E.b, E.c, or E.d); location map bit(s) when the case of cell does not belong to E.d.

Preprocessing. Calculate $A_{1}$ and $A_{2}$ using (14) and (16). For $0 \leq A_{2} \leq 255$, process the verification test as follows.

Calculate the test differences $d_{1}$ and $d_{2}$ :

$$
d_{1}=A_{2}-L_{1}, \quad d_{2}=L_{2}-A_{2} .
$$

Hide the test bits to the test differences $d_{1}$ and $d_{2}$ as follows:

$$
D_{i}= \begin{cases}2 \cdot d_{i}+b_{t} & \text { if } d_{i} \in\left[T_{n} ; T_{p}\right] \\ d_{i}+T_{p}+1 & \text { if } d_{i}>T_{p} \\ d_{i}+T_{n} & \text { if } d_{i}<T_{n}\end{cases}
$$

where $i=1,2 ; D_{1}$ and $D_{2}$ are the modified test differences; $b_{t}$ is the test bit. If $d_{1}$ or $d_{2}$ is negative, $b_{t}$ is 0 ; otherwise, $b_{t}$ is 1 .

Calculate the test pixel values $A^{\prime}$ and $A^{\prime \prime}$ :

$$
A^{\prime}=D_{1}+L_{1}, \quad A^{\prime \prime}=L_{2}-D_{2} .
$$

Define the proper case for the tested cell:

(E.a) if a cell has $A_{1}<0$ or $A_{1}>255$, then we mark the cell as " 1 " in the location map. No bit can be embedded into this cell;

(E.b) if a cell has $0 \leq A_{1} \leq 255$ and $A_{2}<0$ or $A_{2}>255$, then we mark the cell as " 01 ." In this case, only one bit can be hidden during the first stage of embedding;

(E.c) if a cell has $0 \leq A_{1} \leq 255,0 \leq A_{2} \leq 255$, and $A^{\prime}<0$, $A^{\prime}>255$ or $A^{\prime \prime}<0, A^{\prime \prime}>255$, then we mark the cell as " 00 " in the location map. Here, we use a test bit to identify whether the cell is to be skipped or not. In this case, the mark identifies the cell can contain two bits of hidden data;

(E.d) if the cell does not belong to the skipped set after the two-stage data hiding process $\left(0 \leq A^{\prime} \leq 255\right.$ and $0 \leq$ $\left.A^{\prime \prime} \leq 255\right)$, then no marker is used. No marker means two bits of successful data hiding.

Similar to the encoder, there are three possible situations regarding the overflow/underflow problems for the decoder.

\subsubsection{Overflow/Underflow Test for Decoder}

Input. Cell for testing $\left(A_{0}, a_{1}, a_{2}, \ldots, a_{8}\right)$; one or two bits from the location map, if necessary; threshold values $T_{n}$ and $T_{p}$.

Output. Case of the cell (i.e., D.a, D.b, D.c, or D.d).

Preprocessing. For a tested cell, process as follows: assume that $A_{2}=A_{0}$, where $A_{0}$ is the modified central pixel of the tested cell. Process the verification test using (39), (40), and (41). Get test pixel values $A^{\prime}$ and $A^{\prime \prime}$.

Define the proper case for the tested cell:

If a cell has $A^{\prime}<0, A^{\prime}>255$ or $A^{\prime \prime}<0, A^{\prime \prime}>255$, then the cell was marked in the location map.

(D.a) If the first location map bit for current cell is " 1 ," no bit was embedded into this cell. Otherwise, read the second bit of the location map and check (D.b)-(D.c). The cell remains the same as original.

(D.b) If the first and second bits of the location map for the current cell are " 01 ," the current cell was modified during the first stage of embedding. In this case, the cell contains one bit of hidden data.

(D.c) If the first and second bits of the location map for the current cell are " 00 ," the current cell was modified during the first and second stages of embedding. In this case, the cell contains two bits of hidden data.

(D.d) If a cell has $0 \leq A^{\prime} \leq 255$ and $0 \leq A^{\prime \prime} \leq 255$, then the cell was not marked in the location map and was modified during the first and second stages of embedding. In this case, the cell contains two bits of hidden data.

The location map is necessary for recovering data and should be hidden to image as part of payload. The experimental results show that the location map size is almost negligible when compared to full capacity. Fortunately, the location map is not necessary sometimes.

\section{Algorithms for Encoder and Decoder}

The encoder and decoder of the proposed method are presented in Figure 5.

Encoder contains four main steps: "Preparation of data and image (i.e., initialization)," "Definition of threshold values," "Data hiding," and "Embedding header information." 


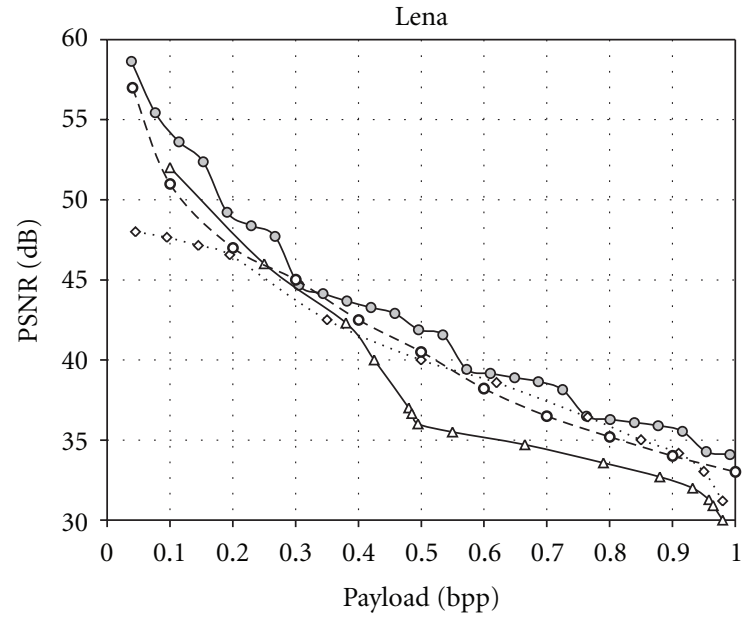

(a)

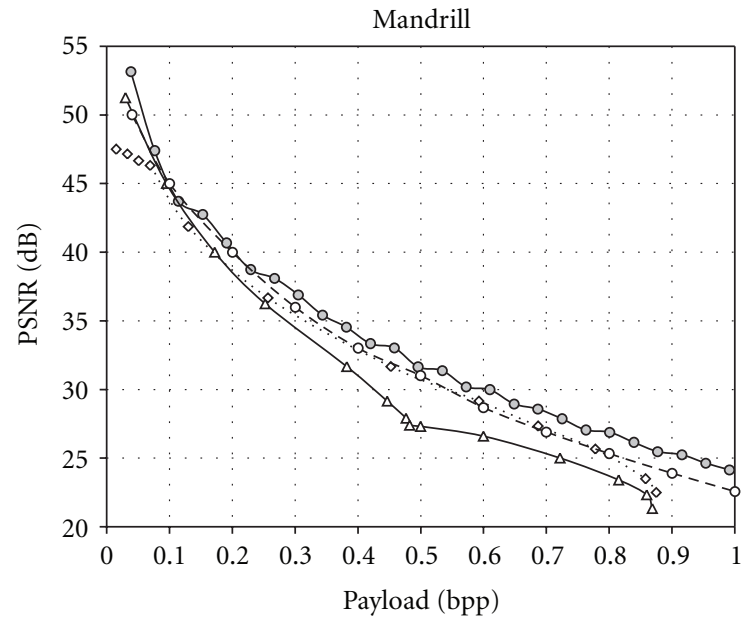

(c)

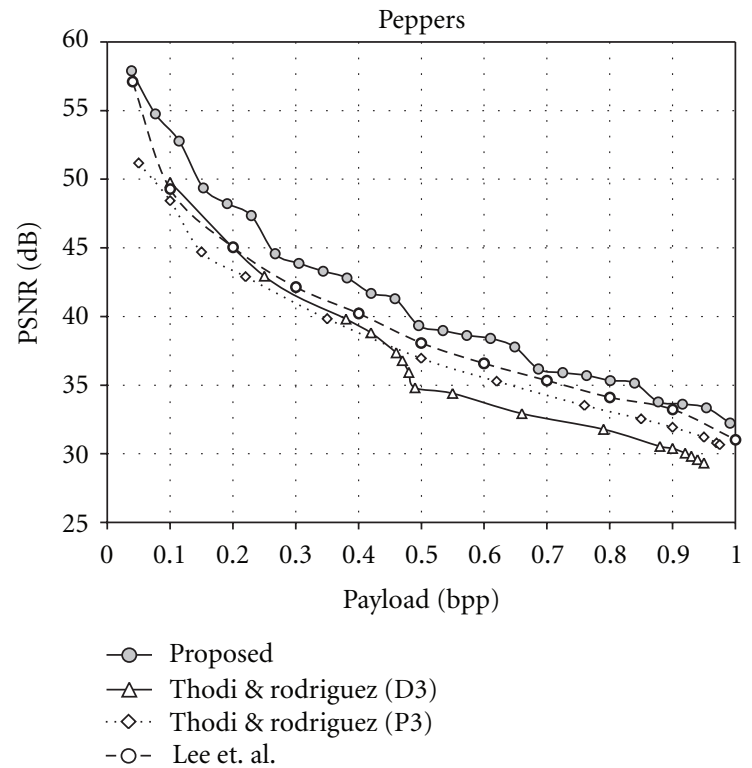

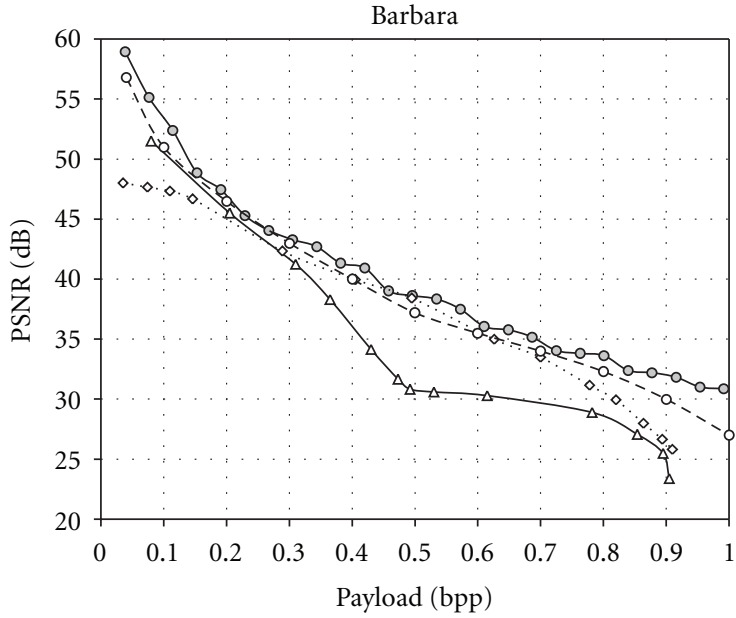

(b)

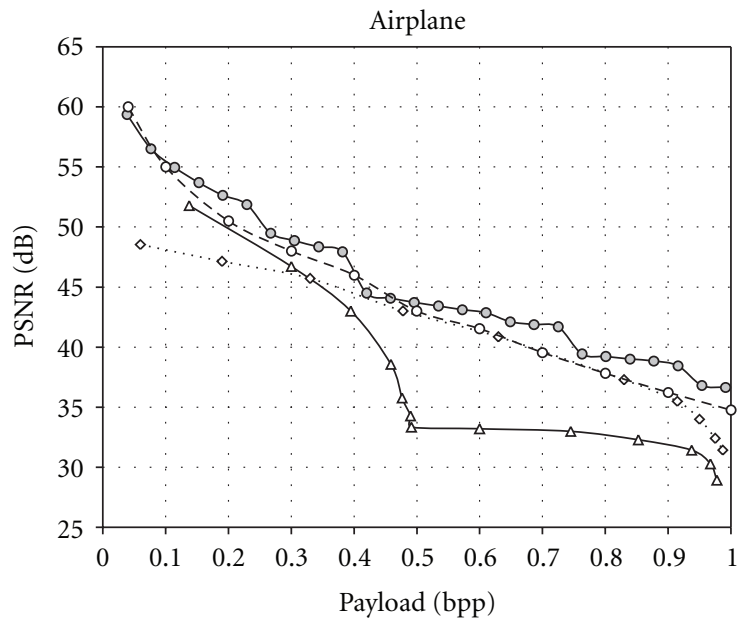

(d)

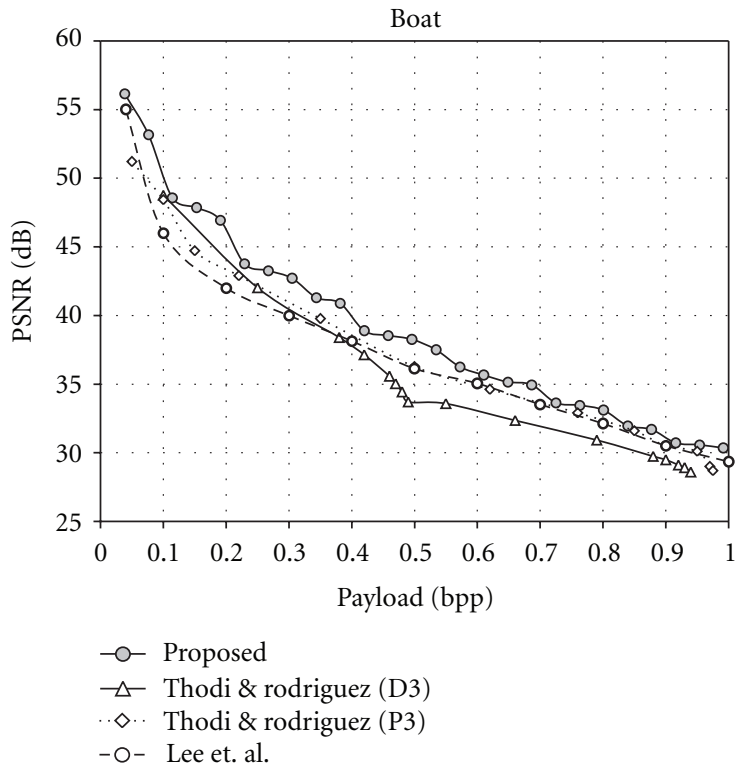

(f)

FIGURE 6: Experimental results. 


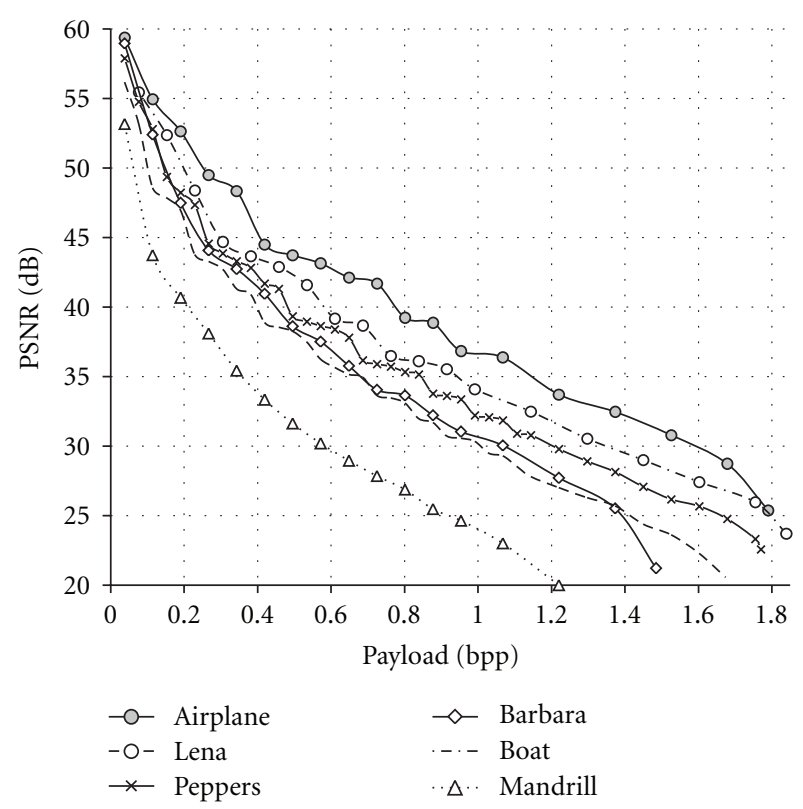

FIGURE 7: Embedding ability of the proposed method.

Step 1 embeds header information into a certain location, and copies the original values and embeds into another location in order to recover the original value. Header information includes a proper payload. Step 2 defines proper threshold values. Step 3 embeds data into image. Here, the block "Update payload I" inserts location map bit (bits) to the first position of the payload stream (i.e., $P=\{L M ; P\}$ ). The block "Update payload II" removes hidden bit (bits) from the payload stream. Note that according to the (13)(16) the proposed method may hide zero, one, or two bits. In case of hiding zero bits, the block "Update payload II" skips updating the payload stream. Data hiding process stops when the last bit from the payload stream $P$ is embedded into the image (i.e., $|P|=0$ ). If the number of the available cells is not enough for hiding payload $P$, increase thresholds values and repeat steps 3 and 4 .

Decoder contains three main steps: "Read header," "Data extraction," and "Recover header and data." Step 1 defines the initial parameters for decoding (threshold values $T_{n}, T_{p}$ and index $i$ ). Cell with index $i$ is the last modified cell in the encoder. Step 2 recovers the hidden payload and the original image. The block "Update payload D" removes the location map bit(bits) from the payload stream $P$. Step 3 recovers the original data where header information is embedded.

\section{Experimental Results}

The proposed two-stage reversible data hiding algorithm is tested over the six well-known uncompressed $512 \times 512$ grayscale images: Lena, Barbara, Mandrill, Peppers, Boat, and Airplane. Performances of the proposed algorithm are compared with well-known methods of Thodi and Rodríguez [14, 19], and Lee et al. [20]. Figure 6 presents the PSNR values for various payloads in different grayscale images. From Figure 6, we can see that the PSNR value of the proposed two-stage embedding is better than that of the existing methods. Particularly in high payloads, the proposed scheme shows better performance. The results clearly indicate the advantage of the two-stage embedding strategy.

For better understanding, the threshold values and squared errors used in our experiments and the respective number of cells in the half-removable and nonremovable sets for Lena image are reported in Table 1. The population of the cells and squared error in each set depends on the type of images and selected threshold values. From Table 1, we can see that the population in the nonremovable set increases with increase in capacity, which makes its performance worse. Since some of the nonremovable cells can be used for data hiding in any one of the stages, the overall performance of the proposed two-stage embedding is better than that of the well-known methods in the literature.

Figure 7 shows the PSNR values for different payloads. From Figure 7, we can see that the maximum payload for Lena, Airplane, Barbara, Peppers, Boat, and Mandrill images is $1.83,1.79,1.48,1.77,1.67$, and $1.21 \mathrm{bpp}$, respectively. We also consider the capacity of the proposed method based on the given minimum allowable distortion. Hence, we select the minimum allowable distortion for each image based on the maximum capacity of the method P3 of Thodi and Rodríguez [14]. In the case of the Airplane image, their maximum payload is $0.98 \mathrm{bpp}$ and its corresponding PSNR value is $32.1 \mathrm{~dB}$. If the $32 \mathrm{~dB}$ is the minimum allowable distortion, our method achieves $1.51 \mathrm{bpp}$, which is 50 percent more than the capacity of the histogram shifting method.

A similar observation can be made about the Barbara, Lena, Peppers, Boat, and Mandrill images. In the case of Mandrill, the maximum capacity is less than the other tested images due to its irregularity in the image, but our capacity is still higher than the existing methods. From the result, we can say that the proposed two-stage embedding algorithm can have lower distortion under the same capacity compared to the existing methods.

\section{Conclusion}

This paper presents a novel two-stage reversible watermarking algorithm with higher capacity and lower distortion. The proposed strategy can embed data twice using the lower and upper bounds computed from the sorted neighboring pixels. The distortion due to embedding data in the first stage can be removed at rare occurrences, mostly reduced, or hardly increased in the second stage. In general, data hiding distorts the original images. Nonremovable case distorts the image like any other methods including histogram shifting approach. Even though the population of the removable case is small, this set never distorts. In case of the halfremovable set, this method distorts less. As a result, this method distorts image less. Also, the problems of overflow and underflow are handled using a special location map similar to the method presented in [19]. Experimental results clearly indicate the advantage of the proposed method versus well-known methods in reversible watermarking in terms of ratio of capacity over distortion. 


\section{Acknowledgments}

This work was supported by the Catholic University of Korea, IT R\&D program (Development of anonymity-based u-knowledge security technology, 2007-S001-01), by the Ministry of Knowledge Economy, Korea, under the ITRC supervised by the National IT Industry Promotion Agency (NIPA-2010-C1090-1001-0004), by the Ministry of Culture, Sports and Tourism and Korea Culture Content Agency in the Culture Technology (CT) Research and Development Program, and by the Ministry of Education, Science and Technology under the supervision of National Research Foundation for 3DLife (FP7). Authors thank the reviewers for their valuable comments which improve the quality of this paper.

\section{References}

[1] F. Mintzer, J. Lotspiech, and N. Morimoto, "Safeguarding digital library contents and users: digital watermarking," $D$ Lib Magazine, vol. 3, no. 12, pp. 33-45, 1997.

[2] J. Fridrich, M. Goljan, and R. Du, "Lossless data embedding for all image formats," in Security and Watermarking of Multimedia Contents IV, vol. 4675 of Proceedings of SPIE, pp. 572-583, January 2002.

[3] M. van der Veen, F. Bruekers, A. Leest, and S. Cavin, "High capacity reversible watermarking for audio," in Security, Steganography, and Watermarking of Multimedia Contents VI, vol. 5020 of Proceedings of SPIE, p. 111, January 2003.

[4] A. Van Leest, M. van der Veen, and F. Bruekers, "Reversible watermarking for images," in Security, Steganography, and Watermaking of Multimedia Contents VI, vol. 5306 of Proceedings of SPIE, pp. 374-385, January 2004.

[5] M. U. Celik, G. Sharma, A. M. Tekalp, and E. Saber, "Reversible data hiding," IEEE International Conference on Image Processing, vol. 2, pp. II/157-II/160, 2002.

[6] B. Yang, M. Schmucker, W. Funk, C. Busch, and S. Sun, "Integer DCT-based reversible watermarking for images using companding technique," in Security, Steganography, and Watermaking of Multimedia Contents VI, vol. 5306 of Proceedings of SPIE, pp. 405-415, January 2004.

[7] B. Yang, M. Schmucker, C. Busch, X. Niu, and S. Sun, "Approaching optimal value expansion for reversible watermarking," in Proceedings of the 7th Multimedia and Security Workshop, pp. 95-101, August 2005.

[8] G. Xuan, J. Chen, J. Zhu, Y. Q. Shi, Z. Ni, and W. Su, "Lossless data hiding based on integer wavelet transform," in Proceedings of the IEEE Workshop on Multimedia Signal Processing, pp. 312-315, St. Thomas, Virgin Island, December 2002.

[9] G. Xuan, Y. Q. Shi, Z. C. Ni et al., "High capacity lossless data hiding based on integer wavelet transform," in Proceedings of the IEEE International Symposium on Circuits and Systems, vol. 2, pp. II29-II32, 2004.

[10] G. Xuan, C. Yang, Y. Zhen, Y. Q. Shi, and Z. Ni, "Reversible data hiding based on wavelet spread spectrum," in Proceedings of the IEEE 6th Workshop on Multimedia Signal Processing, pp. 211-214, Siena, Italy, 2004.

[11] D. Zou, Y. Q. Shi, Z. Ni, and W. Su, "A semi-fragile lossless digital watermarking scheme based on integer wavelet transform," IEEE Transactions on Circuits and Systems for Video Technology, vol. 16, no. 10, pp. 1294-1300, 2006.
[12] L. Kamstra and H. J. A. M. Heijmans, "Reversible data embedding into images using wavelet techniques and sorting," IEEE Transactions on Image Processing, vol. 14, no. 12, pp. 2082-2090, 2005.

[13] H. J. Kim, V. Sachnev, Y. Q. Shi, J. Nam, and H. G. Choo, "A novel difference expansion transform for reversible data embedding," IEEE Transactions on Information Forensics and Security, vol. 3, no. 3, pp. 456-465, 2008.

[14] D. M. Thodi and J. J. Rodríguez, "Expansion embedding techniques for reversible watermarking," IEEE Transactions on Image Processing, vol. 16, no. 3, pp. 721-730, 2007.

[15] J. Tian, "Reversible data embedding using a difference expansion," IEEE Transactions on Circuits and Systems for Video Technology, vol. 13, no. 8, pp. 890-896, 2003.

[16] W. Hong, T. S. Chen, and C. W. Shiu, "Reversible data hiding based on histogram shifting of prediction errors," in Proceedings of the 2nd International Symposium on Intelligent Information Technology Application Workshop (IITA '08), pp. 292-295, December 2008.

[17] H. C. Huang, I. H. Wang, and Y. Y. Lu, "High capacity reversible data hiding with adjacent-pixel-based difference expansion," in Proceedings of the 4th International Conference on Innovative Computing, Information and Control (ICICIC '09), pp. 639-642, December 2009.

[18] Z. Ni, Y. Q. Shi, N. Ansari, W. Su, Q. Sun, and X. Lin, "Robust lossless image data hiding," in Proceedings of the IEEE International Conference on Multimedia and Expo (ICME '04), vol. 3, pp. 2199-2202, Taipei, Taiwan, 2004.

[19] D. M. Thodi and J. J. Rodríguez, "Reversible watermarking by prediction-error expansion," in Proceedings of the 6th IEEE Southwest Symposium on Image Analysis and Interpretation, vol. 6, pp. 21-25, Lake Tahoe, Calif, USA, March 2004.

[20] S. Lee, C. D. Yoo, and T. Kalker, "Reversible image watermarking based on integer-to-integer wavelet transform," IEEE Transactions on Information Forensics and Security, vol. 2, no. 3, pp. 321-330, 2007.

[21] V. Sachnev, H. J. Kim, J. Nam, S. Suresh, and Y. Q. Shi, "Reversible watermarking algorithm using sorting and prediction," IEEE Transactions on Circuits and Systems for Video Technology, vol. 19, no. 7, pp. 989-999, 2009.

[22] A. M. Alattar, "Reversible watermark using difference expansion of triplets," in Proceedings of the International Conference on Image Processing (ICIP '03), pp. 501-504, September 2003.

[23] A. M. Alattar, "Reversible watermark using difference expansion of quads," in Proceedings of the IEEE International Conference on Acoustics, Speech, and Signal Processing, pp. III377-III380, May 2004.

[24] A. M. Alattar, "Reversible watermark using the difference expansion of a generalized integer transform," IEEE Transactions on Image Processing, vol. 13, no. 8, pp. 1147-1156, 2004. 